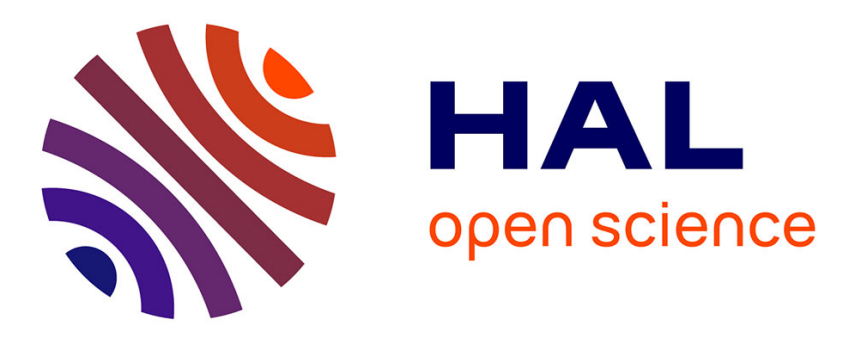

\title{
High Level Synthesis of Globally Asynchronous Locally Synchronous Circuits
}

Christophe Wolinski, Mohammed Belhadj

\section{To cite this version:}

Christophe Wolinski, Mohammed Belhadj. High Level Synthesis of Globally Asynchronous Locally Synchronous Circuits. The Third Annual Atlantic Test Workshop, ATW '94, Jun 1994, Nîmes, France. pp.u-1 - u-4, 10.1109/ATW.1994.747847 . hal-00545571

\section{HAL Id: hal-00545571 \\ https://hal.science/hal-00545571}

Submitted on 10 Dec 2010

HAL is a multi-disciplinary open access archive for the deposit and dissemination of scientific research documents, whether they are published or not. The documents may come from teaching and research institutions in France or abroad, or from public or private research centers.
L'archive ouverte pluridisciplinaire HAL, est destinée au dépôt et à la diffusion de documents scientifiques de niveau recherche, publiés ou non, émanant des établissements d'enseignement et de recherche français ou étrangers, des laboratoires publics ou privés. 


\title{
HIGH LEVEL SYNTHESIS OF GLOBALLY ASYNCHRONOUS LOCALLY SYNCHRONOUS CIRCUITS
}

\author{
Krzysztof WOLINSKI and Mohammed BELHAD.J
}

\author{
IRISA, Campus de Beaulieu \\ 35042 Rennes, FRANCE
}

\begin{abstract}
This paper presents an approach for the design of Globally Asynchronous Locally Synchronous (GALS) circuits. The mixed style using asynchronous and synchronous circuits amalgamates the both styles best features. A language for high level specification of circuits is described. Then, the synthesis method that maps the algorithmic level specification in a net of GALS circuits is given. The asynchronous part is highlighted and avoidance of metastability is described. Finally, the link to existing CAD tools is given via VHDL.
\end{abstract}

\section{Introduction}

Advances in VLSI increese both area and speed of circuits. Easy handling of such circuits depends on the use of design automation tools (e.g. High, Register-Transfer and Logic level synthesis).

Synchronous automatic design tools are widespread and well-known as efficient methodologies. Correct functionalities of a synchronous system depend on the accuracy of the distribution of clock. Many attention in industry and academy has been given to the characteristics of the clock signals [4]. However, as the clock frequency increases, synchronous design becomes more difficult; problems like clock skew, metastabilty increase dramatically. A large part of ICs is devoted to clock generation and buffering.

A promising alternative is the use of asynchronous design where the absence of clock solves those problems, and offers good properties like composability and robustness [3]. But, asynchronous design have also their drawbacks: larger area, rarely mature industrial design tools, etc.

A good compromise seems to be the use of synchronous blocks that communicate by asynchronous techniques. For a discussion on advantages and disavantages of synchronous, asynchronous and mixed styles, see [5].

The work described here emphasizes the aspects of synthesis of Globally Asynchronous Locally Synchronous (GALS) circuits from the high level description language SIGNAL.

The originality of this approach is that the synthesis procedure is build upon the properties of the language. The asynchronous part is built with delay-insensitive elements [8]. This leads to robustness and composability of generated circuits.

The following section describes the input language SIGNAL, and its intermediate form. Then the synthesis method is described. A particular focus will be made in the asychronous part design. Then, a prototype using the Synopsys [1] VHDL synthesis environment is described.

\section{The input language}

Signal is an equational language for the design of reactive applications [6]. It is a formally defined language with a small set of operators.

Signal programs describe relationships between signals (a signal is a stream of typed values). Every signal possesses a clock ${ }^{1}$ which determines if the signal is present or absent $(\perp)$. The Signal kernel is the minimum set of operators with which we can construct any SIGNAL program:

- The usual arithmetic and logic functions

- The $\$$ (delay) operator gives access to the last values of a signal.

- The under-sampling operator allows conditional extraction of values from a given signal: $\mathrm{Y}:=\mathrm{X}$ when $\mathrm{C}$, $\mathrm{Y}$ is equal to $\mathrm{X}$ when the boolean signal $\mathrm{C}$ is TRUE.

$$
\begin{array}{lccccccccc}
\mathrm{X}: & x_{1} & x_{2} & \perp & x_{3} & \perp & x_{4} & x_{5} & x_{6} & \ldots \\
\mathrm{C}: & F & T & F & F & T & T & \perp & T & \ldots \\
\mathrm{Y}: & \perp & x_{2} & \perp & \perp & \perp & x_{4} & \perp & x_{6} & \ldots
\end{array}
$$

- The default operator allows the deterministic merge of signals: $\mathrm{Z}:=\mathrm{X}$ default $\mathrm{Y}, \mathrm{Z}$ merges $\mathrm{X}$ and $\mathrm{Y}$ with priority to $\mathrm{X}$ when both signals are present.

$\begin{array}{ccccccc}\mathrm{X}: & x_{1} & x_{2} & \perp & x_{3} & \perp & \ldots \\ \mathrm{Y}: & y_{1} & \perp & y_{2} & \perp & y_{3} & \ldots \\ \mathrm{Z}: & x_{1} & x_{2} & y_{2} & x_{3} & y_{3} & \ldots\end{array}$

Other operators have been defined using this kernel that permit the reduction of programming effort. For example $\mathrm{Y}:=\mathrm{X} \operatorname{cell} \mathrm{B}$ is the memory operator that can be coded using Signal kernel operators[6].

A Dynamic graph (noted DG) is associated with a SIGNAL program. It describes the dependency of data and the

\footnotetext{
${ }^{1}$ clock is only a logical signal true when a signal is present and absent otherwise
} 
relationship between clocks. The DG of SignaL programs are generated during the compilation process.

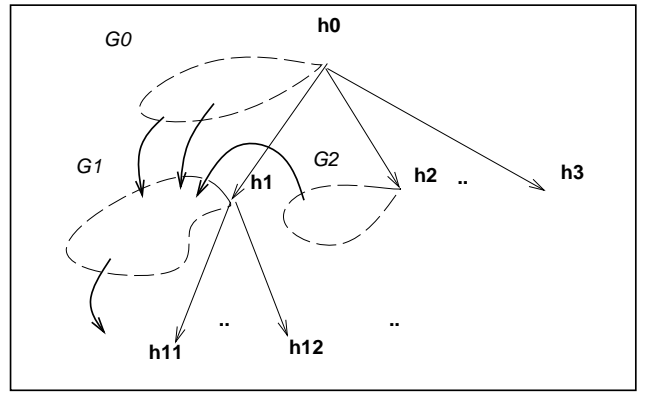

Fig. 1: SignAL internal graph representation

In Fig. $1 h_{0}$ is the fastest clock of the sub-system (in Signal there is no general global clock, the fastest clock is computed for every system). The clocks $h_{1}, h_{2}$ and $h_{3}$ are sub-samplings of $h_{0}$. This means that if $h_{0}$ is absent then $h_{1}, h_{2}$ and $h_{3}$ are absent. This permits the reduction of computation frequency. A conditional data dependency graph is associated with every clock (e.g. $G_{0}$ with $h_{0}$ ). This graph represents all signals computed as frequently as the associated clock.

Let look at the following example to give an insight:

\section{$\mathrm{C}:=(\mathrm{X} 1+\mathrm{X} 2$ when $(\mathrm{A}>\mathrm{B})) \operatorname{default}(\mathrm{X} 3 * \mathrm{X} 4$ when $(\mathrm{A}<=\mathrm{B}))$}

If we suppose that the clock of $\mathrm{A}$ and $\mathrm{B}$ is $h$ (Fig. 2), A value $(a)$ is read when the clock $h$ is true (idem. for B). The operation $x_{1}+x_{2}$ is done only if $h_{1}$ is true (i.e. if $\mathrm{A}>\mathrm{B}$ ). Moreover, if $h$ is absent we do not need to compute $h_{1}$ and $h_{2}$ and their corresponding graphs.

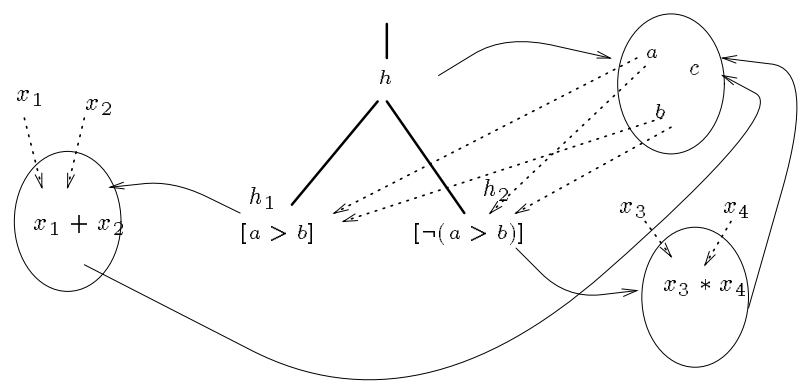

Fig. 2: An example of dynamic Graph

For a formal definition of dynamic garphs, see [6].

\section{Synthesis method}

This section presents how we transform the DG into a net of GALS circuits. By applying some transformations we produce a new graph (a net of processes that we can describe in SignaL). Ultimately a process on the net will correspond to an elementary processor (a GALS circuit).

\section{A. Transformations}

The synthesis process consists of two transformations:
- Construct a net composed of elements that implement the Signal operators deterministic merge, subsampling, etc (we note the implemented operators as: c_or, c_cell, c_when, etc), fork ${ }^{2}$ operators, and communication channels, using direct substitution from DG. This is a direct implementation.

- Partitioning the DG into subgraph containing at most two different clocks. Each subgraph will ultimately correspond to an elemantary processor.

Formally the two transformations corresponds to a closure of the graph. The resulting graph is a net of elementary processors and fork operators connected with channels synchronized by events (an event is the rising or falling edge of a signal).

Intuitively, every SIGNAL operator op can be described as two operations, op and op corresponding to computation of value and clock of the output signal respectively.

$$
c=a \mathrm{op} b \equiv\left\{\begin{array}{l}
c_{v}=a \mathrm{Op}_{v} b \\
c_{h}=a \overline{\mathrm{op}}_{h}^{b} b
\end{array}\right.
$$

where $a \equiv\left\{a_{v}, a_{h}\right\}, a_{v}$ is the value of $a$ and $a_{h}$ represent its clock (true when $a$ is presnt false otherwise). Note here that we have substitute the absence by the value false. To do so, we need a reference clock: the fastest clock of the net. Clock here refers simply to a sequence of edge triggered asynchronous "events" and not to a physical synchronous clock.

Signals in SignAL language are replaced by eventsynchronized channels (hand-shake). A signal $\mathrm{C}$, is defined as follows: $\left\{c_{v}, c_{h v}, c_{h}, c_{h a c k}\right\}$ where $c_{v}$ is the value of the signal $\mathrm{C}$ in terms of the SIGNAL language, $c_{h v}$ is the value of the clock of signal $\mathrm{C}$ (i.e. if $c_{h v}$ is true than $\mathrm{C}$ is present for the current instant of reference clock $c_{h}$, otherwise it is absent), $c_{h}$ represents the reference clock or the fastest clock (for this part of the net). It is represented physically by an event. $c_{\text {hack }}$ is an acknowledgment that corresponds to the end of possible computation for the current tick of $c_{h}$.

So, the opertors are replaced as described in (1), adding a local conditioning mechanism for the op and op computation, and adding a mechanism for the output reference clock computation. The signals are replaced by channels. Finally, the substituted graph is partitioned.

\section{B. Resulting Net}

After the two transformations we obtain a net of processes (Fig. 3), where data and clock transfer use handshake.

The synthesized subgraph (a process or physically a processor) is composed of an asynchronous control part and a synchronous part for the computation of op and

\footnotetext{
${ }^{2}$ fork broadcasts its input signal to a number of outputs
} 
op . The asynchronous part ensures that the computations are done when necessary. The decision is made dynamically (and locally), by considering the values of clock of input channels with respect to the reference clock and the position of the element in the net.

For (Fig. 2) example, the asynchronous part of processors P1 and P2 (Fig. 3) enable computation in synchronous parts if $a_{h v}, a_{h}, a_{v}$ arrive, and $a_{h v}$ is true. Moreover, $(a>b)$ from $\mathrm{P} 1$ and $(a \leq b)$ must be true. If the computation is not necessary and the result of the operation is needed for another computation, we send in the output channel the clock value (hv) false (the value of channel is not important). Note, that the number of request and acknowledge signals are reduced (e.g signals from the same synchronous part of a processor use one request).

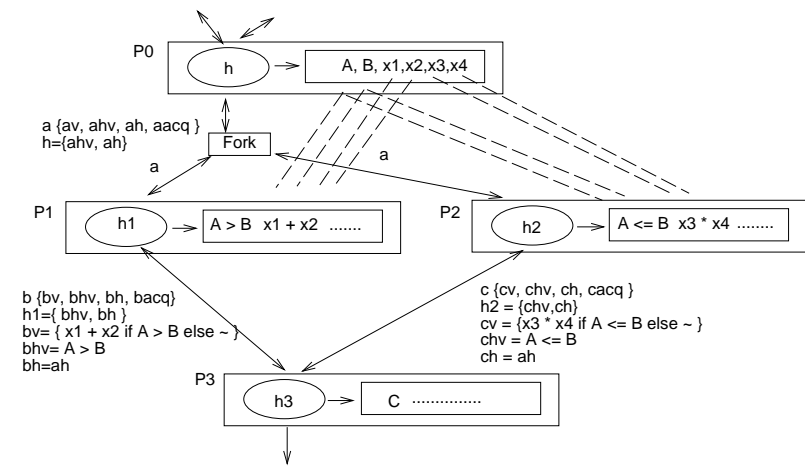

Fig. 3: A transformed Graph

\section{Elementary processor}

An elementary processor (Fig.4) is composed of: input channels and output channels and perhaps, a parallel interface using "hand-shake" that permit the implementation of synchronous procedure call, by sending parameters and receiving results.

An elementary processor is composed of two parts: a synchronous part and an asynchronous one. The asynchronous part (implemented with delay-insensitive elements: select, c-muller, merge, etc [8]) determines when the processor is supposed to compute its results. This decision is taken dynamically. The synchronous part executes the computation corresponding to the reduced part of the net.

The synchronous part can be optimized using classical methods (FSM reduction, hardware sharing, etc).

\section{Asynchronous part}

The asynchronous part (see Fig. 4) of an elementary processor enables the execution of operators op and $\mathrm{op} h_{h}$ corresponding to the computation (associated with this processor), evaluates the reference clock (signals $\mathrm{CH}$, CHV), and generates acknowledgments (Aacq, Bacq).

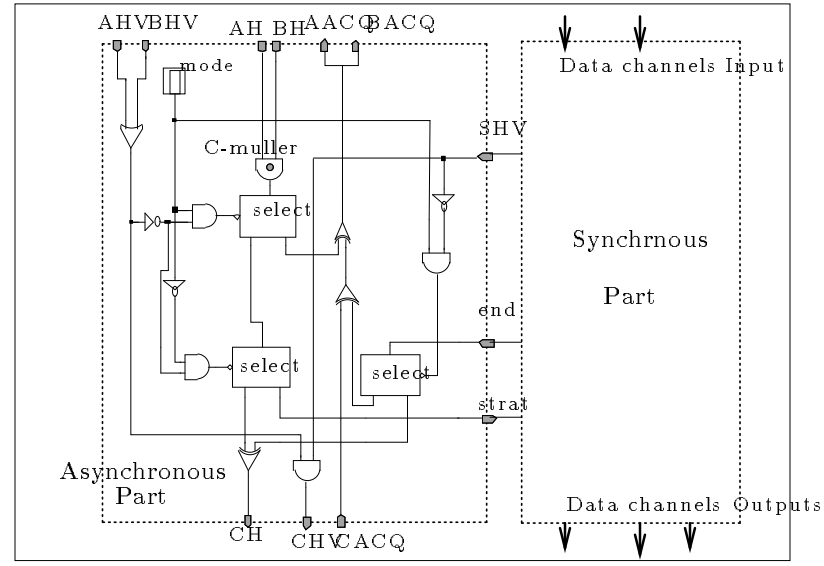

Fig. 4: Elementary processor

The decision of enabling the computation takes into account Ahv, Bhv and position of the processor in the net if the processor outputs are not used as input in other processors optimization on computation frequency is possible).

The value SHV (Fig. 4) is given by the synchronous part. It corresponds to the current value of the clock. The synchronous part is awoken when an event occur in the signal start. There is an end of the computation (an event occurs on end) either when SHV is false or all computations corresponding to the synchronous part are finished.

\section{A. Hand-shaking problems}

Our implementation uses a two phases protocol [8]. The generated architecture must guarantee that a data arrives before its corresponding request signal. Data used by synchronous part are prepared by preceding processors in the net (e.g. Fig. 5 ).

The events $\mathrm{AH}$ and $\mathrm{BH}$ are generated by asynchronous parts of processors $A$ and $B$, after their respective synchronous processors ended their computations. Then, the data must be stable before the asynchronous part of the processor $\mathrm{C}$ generates the computation event.

Without the time corresponding to connections routing delays, the request events (e.g $\mathrm{AH}, \mathrm{BH}$ ) have a delay:

$$
\Delta T=2 T_{\text {osc }}+3 T_{\text {select }}+T_{\text {xor }}+T_{c-\text { muller }}
$$

regarding the end of computation of data $\left(T_{o s c}\right.$ : delay of 1 cycle of physical clock oscillator, $T_{\text {select }}$ : delay of a select operator ...).

The decision taken by the asynchronous part uses the hand-shake signals $\mathrm{AH}, \mathrm{BH}$ and the logical signals $\mathrm{AHV}$ and BHV. The processor $\mathrm{C}$ operates correctly if AHV and $\mathrm{BHV}$ are stable before the arrival of the events $\mathrm{AH}$ and $\mathrm{BH}$ (request). Any asynchronous part ensures the correct behavior (of the hand-shake) because it generates the request signal H (e.g AH,BH,CH) after the signal HV is stable.

If the routing conditions are not arbitrary, the handshaking operates correctly. 


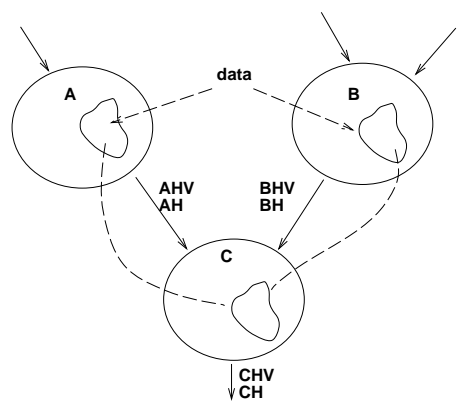

Fig. 5: A part of a net

\section{B. Metastability problems}

The generated architecture being composed of asynchronous and synchronous parts, the question of metastability may arize. The asynchronous part uses delayinsensitive operators but the synchronous part uses normal flip-flop. Previous works [5][7] use special flip-flop called Q-modules to handle the interface between synchronous modules and hand-shake circuits. In the following we describe how the metastability can be avoided in our case.

There are two possible cases for the generation of $\mathrm{CHV}$ and $\mathrm{CH}$ signals:

- No computation is needed: the delay for the computation of CHV (2) is smaller than the delay needed to produce the event $\mathrm{CH}(3)$.

$$
\begin{gathered}
\Delta T_{\text {AHtoCHV}}=T_{o r}+T_{\text {and }}(2) \\
\Delta T_{\text {AHtoC } H}=T_{c-\text { muller }}+2 T_{\text {select }}+T_{\text {xor }}
\end{gathered}
$$

- Computation is necessary: when the computation ends the SHV event is stable and $\mathrm{CH}$ is produced afterwards (4), at the time of the request of the synchronous part of elementary process signal end (5).

$$
\begin{gathered}
\Delta T_{S H V t o C H V}=T_{\text {and }}(4) \\
\Delta T_{\text {end } t o C H}=T_{o s c}+T_{\text {select }}+T_{x o r}(5)
\end{gathered}
$$

The synchronization between asynchronous and synchronous parts is done by a synchronous automaton that samples the signal start. To avoid metastability (or more accurately to minimize it) the sampling is done in the falling edge of the internal physical clock, while the automaton is activated on the rising edge.

\section{Experimental results}

We have synthesized an architecture from a SIGNAL program describing a control process (see [9] for a complete example). The result was described in structural VHDL and was validated under the VHDL simulation environment.

Synchronous parts were automatically synthesized by Synopsys [1], while asynchronous parts were generated separately. An implementation in the Synopsys and Xilinx FPGA [2] environments has been achieved (Fig. 6).

\section{Conclusion and further works}

We propose a general method for the synthesis of GALS circuits from Signal specification. The synthesis procedure transforms the intermediate form into another graph representing the implementation in terms of circuits behavior. The synthesis uses the notion of local clock in SIGNAL which reduces the frequency of computation.

The main advantages are: absence of global clock (no skew problem), dynamic optimization, and access to VHDL synthesis tools and SIGNAL environment (offering possibility for formal proof, simulation, etc). The drawback of this approach is the size of the resulting circuit, we are currently working on the optimization of the synthesis results.

The complete automatic translation from SIGNAL to Xilinx FPGA is under development, and in near future a cell generator for the asynchronous part (in CMOS technology) will be developed. Moreover, a study is conducted separately for generating distributed code for parallel machines using the same partitioning of SIGNAL programs, to permit Hardware/Software codesign.

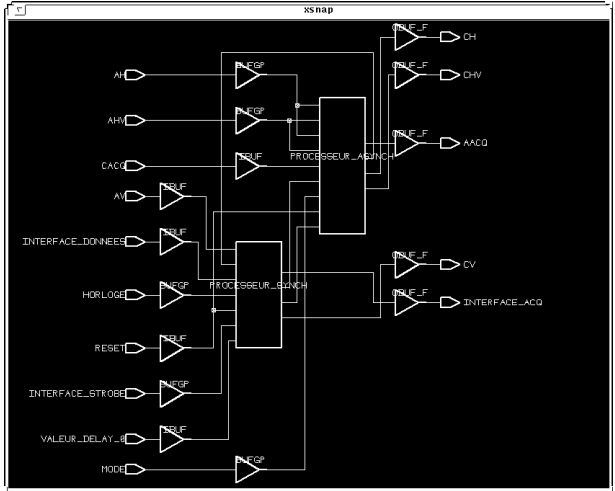

Fig. 6: Xilinx implementation of an elementary processor

\section{References}

[1] Synopsys VHDL Compiler Reference Manual.

[2] The XC4000 Data Book, Programmable Gate array. XILINX, Inc. 1990.

[3] Special issue on Asynchronous systems. Integration, the VLSI journal, 15, 1993.

[4] E. Freidman. Clock distribution design in VLSI circuits- an overview. In IEEE International Symposium on Circuits and Systems, May 1993.

[5] G. Goplalakrishnan and L. Josephson. Towards amalgamating the synchronous and asynchronous styles. In TAU'93, ACM Workshop on Timing Issues in the specification and synthesis of digital systems, 1993.

[6] P. Le Guernic, Th. Gautier, M. Le Borgne, and C. Le Maire. Programming real-time applications with SIGNAL. Proceedings of the IEEE, 79(9):1321-1336, sep 1991.

[7] F. Rosenberger, Ch. Molnar, Th. Chaney and T. Fang. Q-modules: internally clocked delay insensitive modules. IEEE Trans. on Comp., 37(9):1005-1018, Sep 1988.

[8] I. E. Sutherland. Micropipelines. Communication of ACM 32(6):720-738, jun 1989.

[9] K. WOLINSKI and M. BELHADJ. Vers la synthese automatique de programmes SIGNAL. Technical Report 746 , IRISA, 1993.(In French) 